\title{
Collimator design for a clinical brain SPECT/MRI insert
}

\author{
Debora Salvado ${ }^{*}$, Kjell Erlandsson ${ }^{1}$, Alexandre Bousse ${ }^{1}$, Michele Occhipinti $^{2}$, Paolo Busca ${ }^{2}$ Carlo Fiorini², \\ Brian Hutton ${ }^{1}$ \\ From PSMR14: 3rd Conference in PET/MR and SPECT/MR \\ Kos Island, Greece. 19-21 May 2014
}

The Institute of Nuclear Medicine, University College London, London, UK

This project's goal is to design a SPECT insert for a clinical MRI system for simultaneous brain SPECT/MR imaging. We assume the stationary SPECT insert will consist of two rings of $\sim 5 \times 5$-cm SiPM-based detectors insensitive to magnetic fields, with 0.8-mm intrinsic resolution. The maximum diameter is $44.5 \mathrm{~cm}$, the minimum diameter is $33 \mathrm{~cm}$ to accommodate the patient and MRI receive/transmit coil, and the FOV has a $20 \mathrm{~cm}$ diameter.

We have compared eight collimator designs: single-, $2 \times 2-$, $3 \times 3-$ and $5+2 \frac{1}{2}-$ pinhole, and single-, 2-, 3- and 1+21/2-slit slit-slat, where $1 / 2$-pinholes/slits are shared between two detectors. Analytical geometric efficiency was calculated for an activity distribution corresponding to a human brain and a target resolution of $10 \mathrm{~mm}$ FWHM at the centre of the FOV. Noise-free data were simulated with and without depth-of-interaction (DOI) information, and reconstructed for uniform, Defrise, Derenzo, and Zubal brain phantoms. For DOI it is assumed that the crystal's first and second half can be differentiated.

Comparing the multi-pinhole and multi-slit slit-slat collimators, the former gives better reconstructed uniformity and trans-axial resolution, while the latter gives better axial resolution. Although the 2x2-pinhole and 2-slit designs give the highest sensitivities, they result in a sub-optimal utilization of the detector FOV. The best options are therefore the $5+2^{1 / 2}$-pinhole and the $1+2^{1 / 2}$-slit systems, with sensitivities of $4.9^{*} 10-4$ and 4.0*10-4, respectively. The brain phantom reconstructions with multi-pinhole collimator are superior as compared to slit-slat, especially in terms of symmetry and realistic activity distribution. DOI information reduces artefacts and improves uniformity in geometric phantoms, although the difference is small for the brain phantom. These results favour a multi-pinhole configuration.

\section{SpringerOpen ${ }^{\circ}$}

C 2014 Salvado et al; licensee Springer This is an Open Access article distributed under the terms of the Creative Commons Attribution License (http://creativecommons.org/licenses/by/4.0), which permits unrestricted use, distribution, and reproduction in any medium, provided the original work is properly cited. 


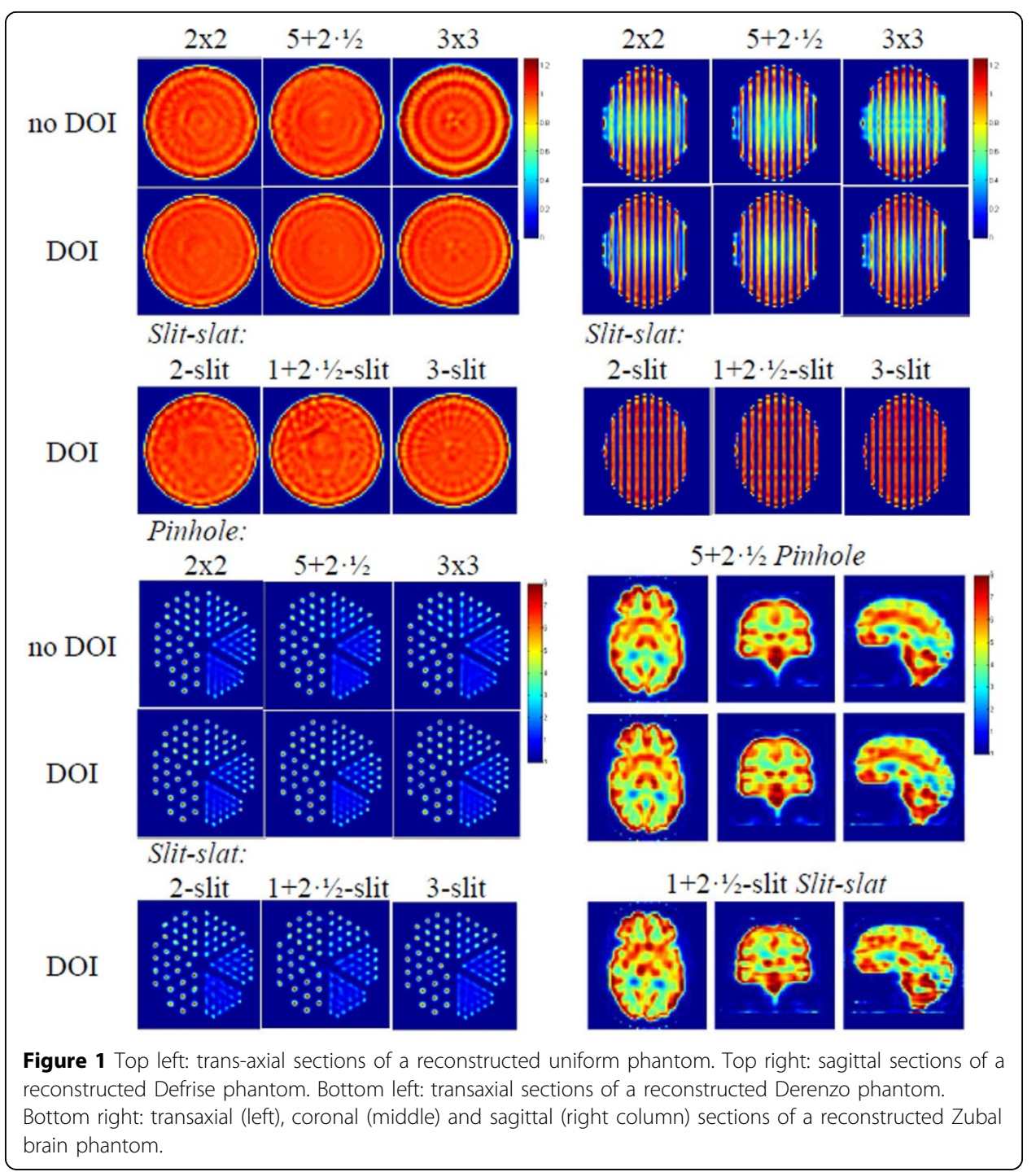

\section{Acknowledgements}

DS is supported by a Portuguese PhD Fellowship (FCT: SFRH/BD/88093/2012). This work was done as part of the INSERT collaboration (FP7-HEALTH-2012-INNOVATION-1: 305311). UCL/UCLH research is supported by the NIHR BRCS funding scheme.

\section{Authors' details}

${ }^{1}$ The Institute of Nuclear Medicine, University College London, London, UK. ²Politecnico di Milano, Milano, Italy.

Published: 29 July 2014

doi:10.1186/2197-7364-1-S1-A21

Cite this article as: Salvado et al.: Collimator design for a clinical brain SPECT/MRI insert. EJNMMI Physics 2014 1(Suppl 1):A21. 